\title{
Nursing Based on Syndrome Differentiation Combined with Auricular Plaster Therapy in Alleviating Insomnia
}

\author{
Ning WANG ${ }^{1}$, Shu-Li MA ${ }^{1, *}, X_{\text {LI }}^{1}$, Li-Xia YANG ${ }^{2}$ \\ ${ }^{1}$ College of Nursing, Shanxi University of Traditional Chinese Medicine,Taiyuan 030024, Shanxi, China; \\ ${ }^{2}$ TCM Department, Shanxi Provincial People’s Hospital, Taiyuan 030012, Shanxi, China
}

\begin{abstract}
Objective: To observe the effect of auricular point pressing combined with nursing based on syndrome differentiation in the treatment of insomnia patients.

Methods: Totally 92 patients with insomnia were randomly divided into the observation groupgiven auricular point pressing combined with nursing care based on syndrome differentiation and the control group treated with auricular point pressing alone. The improvement of Pittsburgh Sleep Quality Index (PSQI), TCM Syndrome Score and nursing satisfaction were compared between the 2 groups.

Results: The time effect of PSQI total score and 6 factors in both groups were statistically significant $(P<0.05)$. The time effect, interaction effect and inter-group effect of subjective sleep quality, PSQI total score were statistically significant $(P<0.05)$; the 4 inter-group factors of sleep quality, sleep latency, sleep duration and daytime function were statistically significant $(P<0.05)$. The total score of TCM syndromes in the observation group was lower than that in the control group $(P<0.05)$, and the satisfaction rate in the observation group higher than that in the control group $(80.43 \%$ vs. $60.86 \%, P<0.05)$.

Conclusion: Auricular point pressing combined withnursing based on syndrome differentiation can effectively improve the sleep quality of insomnia patients, and has significant advantages in improving TCM syndromes, as well as gained higher nursing satisfaction from patients.

Key words: Insomnia; Auricular-plaster therapy/Auricular point pressing; Nursing based on syndrome differentiation/Syndrome differentiation and nursing; Sleep quality; Nursing of integrated traditional Chinese and western medicine
\end{abstract}

\section{Introduction}

Insomnia is mainly manifested as difficulty in falling asleep, or easy to wake up, difficulty to fall asleep after waking up, or even sleepless all night, often accompanied *Corresponding Author:

Shu-Li MA, Master tutor, Shanxi University of Traditional Chinese Medicine, Taiyuan 030024, Shanxi, China E-mail: shuli.ma@I63.com.

Received: March 31 2019, Revised:April 15 2019,Accepted:April 20 2019, Online: May 152019 by headache, dizziness, dreaminess, palpitation, amnesia and other symptoms ${ }^{[1]}$. It can be classified into the following syndrome types: deficiency of heart and spleen, heart-gallbladder qi deficiency, hyperactivity of fire due to yin deficiency, phlegm heatdisturbance, liver depression transforming into fire, Deng et $a l^{[2]}$. Insomnia not only 
affects people's study, life and work, but also increases the risk of cardiovascular diseases, leading to self-disgust, anxiety and depression. As a unique nursing technique of traditional Chinese medicine (TCM), Auricular point pressing, also named auricular plaster therapy, can adjust the balance of yin and yang and regulate the functions of viscera and organs by pressing and stimulating auricular acupoints, thus improving patients' sleep quality ${ }^{[3]}$. Differentiation of symptoms and signs is the essence of TCM nursing. In the nursing of insomnia, after identifying the syndrome types of each insomniac patient, a comprehensive nursing plan is formulated ${ }^{[4]}$. The purpose of this study is to give full play to the advantages of TCM nursing, inherit the TCM non-drug characteristic therapy, according to the syndrome differentiation of insomnia patients, provide the TCM syndrome differentiation nursing scheme for insomnia patients, explore its impact on sleep quality and TCM syndrome, and provide "green and effective" nursing measures for insomnia patients. The results of this study are reported as follows.

\section{Material and Methods}

\section{Study design}

This was a quasi-randomized control trial. The study was divided into groups according to the end of hospitalization number, odd number into control group, even number into observation group, 46 cases in each group.

\section{Participants}

All the 92 insomanics were selected as observation objects who were treated at the Department of traditional Chinese Medicine of Shanxi People's Hospital from February 2018 to January 2019. This study included 26 cases of hypertension, 19 cases of diabetes, 15 cases of coronary heart disease, 13 cases of cervical spondylosis, 11 cases of myocardial infarction and 8 cases of chronic gastritis. There was no significant difference in the general data of gender, age, education level, course of disease, type of disease and syndrome type between the 2 groups $(P>$ 0.05), which was comparable (Table 1).

They all met the following criteria: (1) Conforming to the Classification and Diagnostic Criteria of Mental Disorders in China (CCMD-3-R $)^{[5]}$, the Diagnostic Criteria and Syndrome Types of Insomnia in Internal Medicine of TCM; (2) Aged 18-75 years old; (3) global PSQI score $>7$; (4) signed the written informed consent voluntarily. (5) Consciousness, absence of psychiatric and other organic diseases; (6) No experience on sleep-related or drug-dependent insomnia drugs in the previous week.

Patients were excluded if they: (1) were allergic to tape; (2) had inflammation, eczema, ulcers, frostbite or larger scar tissue in their ears; (3) existed obvious aphasia, aphasia and cognitive impairment. (4) were taking drugs that may affect sleep quality. (5) were family planning,

Table1 Baseline Participant Characteristics ( $n=92)$

\begin{tabular}{|c|c|c|c|c|}
\hline \multirow{2}{*}{ General data } & \multicolumn{2}{|c|}{ Groups } & \multirow{2}{*}{$t / \chi^{2}$} & \multirow{2}{*}{$P$-value } \\
\hline & Control group & Observation group & & \\
\hline Gender $[\mathrm{n}(\%)]$ & & & 0.046 & 0.830 \\
\hline Female & $18(39.1)$ & $17(37.0)$ & & \\
\hline Male & $28(60.9)$ & $29(63.0)$ & & \\
\hline Age (years, $\bar{x} \pm s$ ) & $52.37 \pm 10.37$ & $52.50 \pm 11.70$ & -0.057 & 0.955 \\
\hline Duration of sleep disturbance (years, $\bar{x} \pm s$ ) & $8.07 \pm 4.79$ & $9.32 \pm 3.36$ & -1.450 & 0.150 \\
\hline Education $[\mathrm{n}(\%)]$ & & & 1.077 & 0.584 \\
\hline High school or below & $27(58.7)$ & $25(54.3)$ & & \\
\hline Junior college degree & $11(23.9)$ & $9(19.6)$ & & \\
\hline Bachelor degree or above & $8(17.4)$ & $12(26.1)$ & & \\
\hline Syndrome types $[\mathrm{n}(\%)]$ & & & 0.445 & 0.801 \\
\hline Liver depression transforming into fire & $21(46.7)$ & $18(39.1)$ & & \\
\hline Deficiency of heart and spleen & $15(32.6)$ & $16(34.8)$ & & \\
\hline Hyperactivity of fire due to yin deficiency & $10(21.7)$ & $12(26.1)$ & & \\
\hline
\end{tabular}


pregnancy, lactation and menstruation during treatment.

Standard of suspension and abscission: (1) adverse reactions such as auricular allergy during intervention; (2) using of drugs or therapies that may interfere with the results of the study during the intervention; (3) not suitable for continuing participation in research because of occurring serious complications and accidents during treatment; (4) interruption or withdrawal of treatment for various reasons; (5) poor compliance and non-cooperation in treatment.

\section{Interventions}

Auricular point pressingselection: the main acupoints are Shenmen, sympathetic autonomic, subcortex, and according to the syndrome differentiation and nursing, points were matched. Liver, pancreas and gallbladder were matched for patients with liver depression transforming into fire. Spleen and heart were matched for patients with heart and spleen deficiency. Heart and kidney were matched for patients with hyperactivity of fire due to yin deficiency. Patients were instructed to press the seeds with appropriate strength until a slight soreness was felt on the points. This was randomly performed 3 times during the day and each time for 3 to 5 minutes, with emphasis on bedtime pressing. It is advisable for patients to feel fever, distension and slight pain.Both ears were used alternately to avoid skin lesion caused by longtime adhesion. Auricular pressing replacement occurred the next day. Subjects were given the treatment 2 weeks per course for 2 consecutive courses.

In the control group, on the basis of routine nursing, intervention of auricular point pressing was given. During the treatment, health education related to insomnia was carried out to explain the relevant knowledge of insomnia, including life habits, dietary habits and emotional considerations. It's necessary to create a good hospitalization environment, care for patients, closely observe patients'emotional changes, communicate with patients, find out patients' problems and worries, and timely help patients to solve, inform patients of the bad life and sleep habits that lead to insomnia, and avoid vigorous activities to promote sleep.
In the observation group, participants was given syndrome differentiation and nursing on the basis of the control group. Dialectical protection is as follows:

is Liver depression transforming into fire: The principles of nursing are soothing the liver and purging fire, clearing the brain and tranquilizing the mind. Living care: spacious living room, dim and comfortable light, suitable temperature and humidity, and participate in some outdoor social activities. Dietary care: Eat more grapefruit, kumquat, white radish, coriander and other products to regulate qi and relieve depression. Eat less food with astringency and acerbity, which can easily aggravate the expression of depression. Recommendation: Sharen Chen Pi Crucian Carp Soup, Sanhua Tea. Emotional conditioning: This type of patients with irritable personality are due to visceral dysfunction caused by emotional changes, so we should help patients to take effective ways to alleviate bad mood. Exercise Guidance: Patients should be instructed to use high-intensity and heavyload exercise as an abdominal exercise. At the same time, patients can do Qigong or Tai Chi to regulate qi and blood.

Deficiency of heart and spleen: The principle of nursing is to nourish the heart and spleen, nourish the heart and calm the mind. Living care: Sleep environment should be quiet and comfortable, avoid strong light stimulation and noise, develop good sleep habits, do not use too much brain before sleep, avoid over-thinking, avoid engaging in tense and exciting activities. Dietary care: You can eat more sweetscented longan, coix seed, red beans, lotus seeds and other products to invigorate the spleen and nourish the heart, avoid fat meat, tobacco, alcohol and other food that moisturize and trap the spleen. Emotional nursing: Most patients of this type have insufficient qi and blood, and lack of security. They were also calm, introverted personality, uneasy mind and overthinking. They should be encouraged and comforted to eliminate their concerns to make them feel warm. Sports guidance: Encourage patients to participate in collective activities, exercise appropriately, for 
example playing Tai Chi that belong to traditional health care skills, in order to enhance their physique.

is Hyperactivity of fire due to yin deficiency: The principle of nursing is to nourish yin and reduce fire, and to calm the mind. Living care: Keep the living environment clean, temperature and humidity appropriate to alleviate the discomfort of patients. Inform patients about the loss of kidney yin by staying up late, so as to guide patients to sleep on time as far as possible. Dietary care: Tell patients can eat more tremella, lily, Zhimu porridge and other products to nourish yin and clear fire, eat less spicy, stimulating food, such as green onion, ginger, garlic, etc., to prevent hot injury body fluid consumption. Emotional care: Most patients with this type of disease have high heart and liver fire and are upset. We should strengthen the psychological counseling of patients and listen to some soothing and quiet music for patients. Exercise guidance: Give the patients the guidance of exercise mode and time. Patients can mainly walk and jog. At the same time, they can carry out traditional Chinese medicine treatment such as Baduanjin, Taijiquan. It is appropriate for patients to playing Tai Chi and Baduanjinwhen the yang qi is rising in the morning.

\section{Outcome measurement}

Primary outcome: The primary outcome of this study is the changes in the Pittsburgh Sleep Quality Index (PSQI) that was developed by Buysse ${ }^{[6]}$. The PSQI is an effective self-report questionnaire used to assess the quality of sleep and sleep disorders. The PSQI contains 19 items including the following seven domains: subjective sleep quality, sleep latency, sleep duration, habitual sleep efficiency, sleep disturbances, sleep medication, and daytime dysfunction. The higher the score, the worse the sleep quality. Because this study was not included in the use of sleeping drugs, factor sleeping drugs were not scored. $\mathrm{Liu}^{[7]}$ and colleagues tested the reliability and validity of the Chinese version of the PSQI score and considered it suitable for Chinese population. The study of $\mathrm{Lu}^{[8]}$ shows that PSQI is also suitable for evaluating the effectiveness of TCM treatment. The PSQI was used to assess sleep quality before, after 4-week and 8-week treatment.
Secondary outcome: Secondary outcome measures contain the TCM symptom score and nursing satisfaction.

is Scoring criteria for TCM syndromes: Referring to the Guiding Principles for Clinical Research of New Chinese Medicine (Treatment of Insomnia), the symptoms in the scoring table are divided into 4 grades: none, mild, moderate and severe according to the degree of severity, which are respectively divided into " 0 ", "1", " 2 " and " 3 ". The tongue image is divided into "none" and "you" two levels, which are respectively divided into " 0 " and " 1 ". The scale was used to evaluate the changes of symptoms related to insomnia of different syndromes before and after 4 weeks of intervention.

is Nursing satisfaction: After 4 weeks of intervention, 2 groups of patients were evaluated for nursing satisfaction, which was divided into four levels: very satisfied, satisfied, general and unsatisfactory.

\section{Statistical analysis}

According to the different observation indexes and data, SPSS23.0 data statistics software package was used for data input and analysis. Before the intervention, the general data of the 2 groups were compared. The counting data were tested by $C h i$-square test and the measurement data were tested by $t$-test. The scores of PSQI before and after the intervention were compared by repeated measurement analysis of variance or $t$-test. $t$-test was used to compare the scores of TCM syndromes between groups and within groups. $P<0.05$ was considered as statistically significant.

\section{Results}

\section{PSQI scores}

Table 2 presented PSQI data for observation and control groups after 4 and 8 weeks of intervention. There was no significant difference in PSQI total score and 6 factor score between the observation group and the control group before intervention $(P>0.05)$. By repeated measurement variance analysis, the total PSQI score and the time effect of six factors in both groups had statistical significance 
$(P<0.05)$. The treatment of the 2 groups improved sleep, and the follow-up effect was good with longterm and medium-term effect. Subjective sleep quality, time effect of PSQI total score, interaction effect and inter-group effect had statistical significance $(P<0.05)$. Subject sleep quality, sleep latency, sleep duration had statistical significance between 2 groups $(P<0.05)$. Inter- day and inter-day functions were significantly affected by 4 factors, indicating that there were differences in the improvement of these 4 factors between the 2 groups by different intervention methods.

\section{TCM symptom score}

Table 3 presents TCM Syndrome Scores for observation

Table 2 PSQI score comparison before, after 4-week and 8-week treatment (points, $\bar{x} \pm s$ )

\begin{tabular}{|c|c|c|c|c|c|c|c|}
\hline PSQI comp onent & Groups & Baseline & $\begin{array}{c}4 \text { week } \\
\text { post-treatment }\end{array}$ & $\begin{array}{c}8 \text { week } \\
\text { post-treatment }\end{array}$ & $F_{\text {time }}$ & $F_{\text {inter-group }}$ & $F_{\text {interaction }}$ \\
\hline \multirow[t]{4}{*}{ Global PSQI score } & Control group & $13.217 \pm 2.159$ & $8.870 \pm 1.694$ & $7.783 \pm 1.896$ & $505.359^{\#}$ & $15.157^{\#}$ & $6.659^{\#}$ \\
\hline & Observation group & $12.957 \pm 1.837$ & $7.413 \pm 1.599$ & $6.152 \pm 1.398$ & & & \\
\hline & $t$ & 0.624 & 4.239 & 4.694 & & & \\
\hline & $P$ & 0.534 & 0.000 & 0.000 & & & \\
\hline \multirow[t]{4}{*}{ Subjective sleep quality } & Control group & $2.348 \pm 0.528$ & $1.435 \pm 0.544$ & $1.152 \pm 0.666$ & $175.909^{\#}$ & $15.569^{\#}$ & $3.270^{*}$ \\
\hline & Observation group & $2.304 \pm 0.591$ & $1.087 \pm 0.354$ & $0.739 \pm 0.492$ & & & \\
\hline & $t$ & 0.373 & 3.635 & 3.386 & & & \\
\hline & $P$ & 0.710 & 0.000 & 0.001 & & & \\
\hline \multirow[t]{4}{*}{ Sleep latency } & Control group & $2.457 \pm 0.689$ & $1.543 \pm 0.504$ & $1.370 \pm 0.572$ & $140.083^{\#}$ & $4.879^{*}$ & $1.230^{\nabla}$ \\
\hline & Observation group & $2.391 \pm 0.682$ & $1.370 \pm 0.572$ & $1.065 \pm 0.389$ & & & \\
\hline & $t$ & 0.456 & 1.548 & 2.985 & & & \\
\hline & $P$ & 0.650 & 0.125 & 0.004 & & & \\
\hline \multirow[t]{4}{*}{ Sleep duration } & Control group & $2.087 \pm 0.725$ & $1.391 \pm 0.537$ & $1.283 \pm 0.502$ & $99.477^{\#}$ & $4.696^{*}$ & $1.060^{\nabla}$ \\
\hline & Observation group & $2.022 \pm 0.649$ & $1.152 \pm 0.469$ & $1.043 \pm 0.363$ & & & \\
\hline & $t$ & 0.454 & 2.274 & 2.620 & & & \\
\hline & $P$ & 0.651 & 0.025 & 0.010 & & & \\
\hline \multirow[t]{4}{*}{ Habitual sleep efficiency } & Control group & $2.500 \pm 0.506$ & $1.609 \pm 0.537$ & $1.370 \pm 0.488$ & $202.879^{\#}$ & $3.333^{\nabla}$ & $0.738^{\nabla}$ \\
\hline & Observationgroup & $2.435 \pm 0.583$ & $1.457 \pm 0.585$ & $1.152 \pm 0.363$ & & & \\
\hline & $t$ & 0.573 & 1.300 & 2.424 & & & \\
\hline & $P$ & 0.568 & 0.197 & 0.017 & & & \\
\hline \multirow[t]{4}{*}{ Sleep disturbances } & Control group & $1.696 \pm 0.511$ & $1.348 \pm 0.525$ & $1.196 \pm 0.453$ & $50.782^{\#}$ & $1.873^{\nabla}$ & $2.048^{\nabla}$ \\
\hline & Observation group & $1.717 \pm 0.584$ & $1.130 \pm 0.341$ & $1.109 \pm 0.315$ & & & \\
\hline & $t$ & -0.190 & 2.354 & 1.069 & & & \\
\hline & $P$ & 0.850 & 0.021 & 0.288 & & & \\
\hline \multirow[t]{4}{*}{ Daytime dysfunction } & Control group & $2.130 \pm 0.749$ & $1.565 \pm 0.544$ & $1.391 \pm 0.493$ & $83.681^{\#}$ & $7.741^{\#}$ & $2.894^{\nabla}$ \\
\hline & Observation group & $2.087 \pm 0.626$ & $1.217 \pm 0.593$ & $1.043 \pm 0.469$ & & & \\
\hline & $t$ & 0.302 & 2.932 & 3.464 & & & \\
\hline & $P$ & 0.763 & 0.004 & 0.001 & & & \\
\hline
\end{tabular}

Notes: ${ }^{*} P<0.05 ;{ }^{\#} P<0.01 ;{ }^{\nabla} P>0.05$. 
and control groups after 4 weeks of intervention.There was no significant difference in the score of syndromes between the 2 groups before intervention $(P>0.05)$, which was comparable. There were significant differences between the 2 groups in improving insomnia symptoms of deficiency of heart and spleen, such as difficulty falling asleep, food less anorexia, dreaminess and wakefulness $(P<0.05)$. There were significant differences between the 2 groups in improving insomnia symptoms of liver depression transforming into fire, such as irritability, food less anorexia, thirst with liking for fluids $(P<0.05$, or $P<0.01)$. There were significant differences between the 2 groups in improving insomnia symptoms of yin-deficiency and fire-exuberance type such as vexation, less dry mouth and body fluid, and irritability $(P<0.05)$.

\section{Nursing satisfaction}

Table 4 presents nursing satisfaction for observation and control groups after 4 weeks of intervention. The satisfaction rate of the observation group was significantly higher than that of the control group, and the difference was statistically significant $(P<0.05)$.

\section{Discussion}

In recent years, many literatures had confirmed that auricular point pressing can effectively improve sleep quality $^{[9-11]}$. The results of PSQI in this study also confirmed the effectiveness of auricular point pressing using wang Buliuxangzi (seeds of semen vaccariae) on improving sleep quality. The time effect of the control

Table 3 Comparison of TCM syndrome scores (points, $\bar{x} \pm s$ )

\begin{tabular}{|c|c|c|c|c|c|}
\hline \multirow[b]{2}{*}{ Syndrome types } & \multirow[b]{2}{*}{ Symptoms } & \multicolumn{2}{|c|}{ Control group } & \multicolumn{2}{|c|}{ Observation group } \\
\hline & & Baseline & $\begin{array}{c}4 \text { weeks } \\
\text { post-treatment }\end{array}$ & Beseline & $\begin{array}{c}4 \text { weeks } \\
\text { post-treatment }\end{array}$ \\
\hline \multirow{3}{*}{$\begin{array}{l}\text { Deficiency of heart } \\
\text { and spleen }\end{array}$} & Difficulty falling asleep & $2.20 \pm 0.414$ & $1.27 \pm 0.594^{\nabla \nabla}$ & $1.81 \pm 0.655$ & $0.81 \pm 0.544^{\nabla \nabla \boldsymbol{\Lambda}}$ \\
\hline & Dreaminess & $1.87 \pm 0.516$ & $1.27 \pm 0.458^{\nabla \nabla}$ & $1.88 \pm 0.619$ & $0.81 \pm 0.544^{\nabla \nabla \boldsymbol{\Lambda}}$ \\
\hline & Wakefulness & $2.13 \pm 0.743$ & $1.13 \pm 0.352^{\nabla \nabla}$ & $1.94 \pm 0.772$ & $0.75 \pm 0.577^{\nabla \nabla \boldsymbol{\Lambda}}$ \\
\hline \multirow{3}{*}{$\begin{array}{l}\text { Liver depression } \\
\text { transforming into fire }\end{array}$} & Irritability & $1.95 \pm 0.669$ & $1.29 \pm 0.463^{\nabla \nabla}$ & $1.94 \pm 0.639$ & $0.94 \pm 0.416^{\nabla \nabla \boldsymbol{\Lambda}}$ \\
\hline & Poor appetite & $2.10 \pm 0.436$ & $1.86 \pm 0.378$ & $2.00 \pm 0.485$ & $0.83 \pm 0.618^{\nabla \nabla \Delta \Lambda}$ \\
\hline & Thirst with liking for fluids & $1.05 \pm 0.740$ & $0.95 \pm 0.498$ & $1.06 \pm 0.539$ & $0.50 \pm 0.514^{\nabla \nabla \boldsymbol{\Lambda}}$ \\
\hline \multirow{3}{*}{$\begin{array}{l}\text { Hyperactivity of fire } \\
\text { due to yin deficiency }\end{array}$} & Vexation & $1.70 \pm 0.483$ & $1.27 \pm 0.422^{\nabla}$ & $1.67 \pm 0.492$ & $0.67 \pm 0.492^{\nabla \nabla \boldsymbol{\Lambda}}$ \\
\hline & Irritability & $1.50 \pm 0.527$ & $1.30 \pm 0.675$ & $1.42 \pm 0.515$ & $0.75 \pm 0.452^{\nabla \nabla \boldsymbol{\Lambda}}$ \\
\hline & $\begin{array}{l}\text { Less dry mouth and body } \\
\text { fluid }\end{array}$ & $1.80 \pm 0.789$ & $1.20 \pm 0.422$ & $1.50 \pm 0.674$ & $0.58 \pm 0.515^{\nabla \nabla \wedge}$ \\
\hline
\end{tabular}

Notes: $t$-test showed that ${ }^{\nabla}$ was $P<0.05$, or ${ }^{\nabla \nabla}$ was $P<0.01$, with significant difference when compared with treatment before. After intervention, the scores of TCM syndromes between the 2 groups were compared, and $t$-test showed that

$\triangle$ was $P<0.05$ and ${ }^{\wedge}{ }^{\star}$ was $P<0.01$.

Table 4 Comparisons of patients' satisfaction between 2 groups [n(\%)]

\begin{tabular}{lcccccc}
\hline \multicolumn{1}{c}{ Groups } & N & Very satisfied & Satisfied & General & Unsatisfactory & Satisfaction rate \\
\hline Observation group & 46 & $22(47.82)$ & $15(32.61)$ & $7(15.22)$ & $2(4.34)$ & $37(80.43)$ \\
\hline Control group & 46 & $16(34.78)$ & $12(26.09)$ & $13(28.26)$ & $5(10.87)$ & $28(60.86)$ \\
\hline
\end{tabular}


group was statistically significant $(P<0.01)$. After 4 weeks and 8 weeks of intervention, the scores of 6 factors were lower than those before intervention, and the difference was statistically significant $(P<0.05)$; the subjective sleep quality and sleep quality after 8 weeks of intervention were significantly lower than those before intervention $(P<0.05)$. The scores of sleep efficiency and daytime dysfunction were lower than those after 4 weeks of intervention $(P<0.05)$. From this study, Auricular point pressing has the following functions: (1) It can effectively improve the insomnia patients with deficiency of heart and spleen, such as difficulty in falling asleep, dreaminess and wakefulness symptoms. (2) It can improve the symptoms of irritability, poor appetite and thirst with liking for fluids in insomnia of liver depression transforming into fire. (3) It can also improve the symptoms of irritability, vexation and less dry mouth and body fluid in insomnia of hyperactivity of fire due to yin deficiency. (4) It can balance yin and yang and regulate the functions of viscera by pressing and stimulating auricular acupoints, thus achieving the effect of treating both symptoms and organs. "Lingshu·Oral Instruction"said that"the ear is the place where the lineage gathers." $\mathrm{Liu}^{[12]}$ found that the stimulation of auricular point pressing can lead to the sensory transmission of 14 meridians. In addition, modern medical studies believe that there are a large number of nerves on human ears. Appropriate stimulation of auricular point pressing can regulate nerve function ${ }^{[13]}$.

Syndrome differentiation and nursing is guided by the traditional basic theory of TCM. Through the data collected from the 4 TCM diagnostic methods of inspection, listening and smelling, inquiry, pulse-taking, we can understand a disease as a whole, identify the etiology and location of the disease, make a diagnosis of "syndrome", and then implement the corresponding nursing interventions according to the results of differentiation of symptoms and signs ${ }^{[14-15]}$. Research shows that the occurrence of insomnia is closely related to living environment, diet, emotions and other factors, while TCM syndrome differentiation and nursing is to give comprehensive nursing plan on the basis of differentiation of symptoms and signs, considering various related factors comprehensively.
In this study, the scores of subjective sleep quality, sleep duration and daytime dysfunction in the observation group after 4 weeks of intervention were lower than those in the control group $(P<0.05)$, and the total PSQI scores, subjective sleep quality, sleep latency, sleep duration, daytime function and sleep efficiencyscores in the observation group after 8 weeks of intervention were lower than those in the control group $(P<0.05)$. In the improvement of TCM syndrome score, auricular point pressing combined with nursing based on syndrome differentiation is superior to single auricular point pressing in improving symptoms of insomnia patients with deficiency of heart and spleen, such as difficulty in falling asleep, dreaminess and wakefulness, is superior in improving symptoms of insomnia patients with insomnia of liver-depression-fire type, such as irritability, poor appetite and thirst with liking for fluids, and is also superior in improving symptoms of insomnia patients with insomnia of hyperactivity of fire due to yin deficiency, such as irritability, vexation and less dry mouth and body fluid. The nursing satisfaction of 2 groups of patients with auricular point pressing combined with syndrome differentiation nursing group was significantly better than that of single auricular point pressing $(P<0.05)$. It shows that auricular point pressing combined with TCM syndrome differentiation and nursing can effectively improve the sleep quality and TCM syndromes of insomnia patients, and the patients'nursing satisfaction is higher. Perfect evaluation is the premise of implementing nursing based on syndrome differentiation, including physical characteristics, daily life, eating habits, sleep status evaluation, and so on. We should evaluate the relationship between sleeping time, waking time and 12 Earthly Branches of TCM, viscera, sleep quality and dreams, solar terms of insomniacs patients, and observes the effect of nursing interventions and sleep situation at any time in order to adjust nursing plan in time. To adapt to the four seasons, according to the particularity of the environment and human body, we can give patients living guidance. According to the homology of medicine and food, the nature of food, we can individualize dietary care for patients according to different people and different syndromes ${ }^{[16]}$. According to the relationship between emotions and viscera, qi and blood, we should 
pay attention to each patient's life experience to provide patients with appropriate emotional care. According to the patient's syndrome type, physical condition, we can give exercise guidance suitable for patients to improve the ups and downs bias of human viscera to better reflect the concept of "harmony between man and nature" of TCM, and better improve the sleep quality of insomniacs.

In summary, we derived the following conclusion: auricular point pressing combined with nursing based on syndrome differentiation of TCM can improve sleep quality and TCM syndromes, and enhance patients' nursing satisfaction.

\section{Declaration}

The authors of this article declare no conflict of interest.

\section{Foundation Item}

The study was approved by Shanxi Graduate Education Innovation Project (2018SY095).

\section{References}

1 China Sleep Research Association. Guidelines for the Diagnosis and Treatment of Insomnia in China. Chin J Med, 2017, 97(24): 1844-1855.

2 Deng Aijun, Jiang Ruixue, Ma Zuofeng. Literature study on syndrome types and distribution characteristics of syndrome elements in insomnia. Modern Dist Educ Chin Med, 2015, 13(7): 147-149.

3 Meng Hui, Zhang Zhigang, Bu Chunlei, et al. Research progress of auricular acupoint pressing therapy for insomnia in recent five years. Sichuan Mental Health, 2017, 30(4): 389-392.

4 Zhao Xiuling. Talking about TCM nursing of insomnia patients. Guangming TCM, 2015, 30(12): 2674-2675.

5 Branch of Psychiatry, Chinese Medical Association. CCMD-3 Classification and Diagnostic Criteria of Mental Disorders in China. 3 Edition. Jinan: Shandong Science and Technology Publishing House, 2001: 118-119.

6 Buysse DJ, Reynolds C, Monk TH, et al. The Pittsburgh Sleep Quality Index: a new instrument for psychiatric practice and research. Psychiatry Res, 1989, 28(2): 193-213.

7 Liu Xianchen, Tang Maoqin. Reliability and validity of Pittsburgh Sleep Quality Index. Chin J Psychiatry, 1996, 29(2): 103-107.

8 Lu Taoying, Li Yan, Xia Ping, et al. Reliability and validity of Pittsburgh Sleep Quality Index. Chongqing Med Coll, 2014, 43(3): 260-263.

9 Yang Yuanyuan, Wang Yan, Li Wenwen, et al. Metaanalysis of the efficacy of auricular point sticking on insomnia. J Nursing, 2015, 30(5): 4-8.

10 Ye Yin, Ding Yulan, Pang Shuqin, et al. Research progress of auricular acupoint pressing with Wang Buliuxingzi in the treatment of post-stroke insomnia. Gen Nurs, 2017, 15(20): 2462-2464.

11 Fan Enfang, Ma Ying. Research progress of ear acupoint sticking therapy for insomnia. Hebei Trad Chin Med, 2014, 36(6): 930-932.

12 Liu Weizhou, Yang Biyun, Li Dingzhong. Single-blind and double-blind observation of sensory transmission of the ear acupoints through the channels. Acupunct Study, 1986, (2): 82-85.

13 Zhang Qibing. Morphological mechanism of ear acupuncture. Shanghai J Acupunct Moxibust, 2003, 22(1): 46-48.

14 Tan Liuchun, Xu Junrong. Summary of clinical application of TCM syndrome differentiation nursing. Gen Nurs, 2011, 9(7): 1964-1965.

15 Chen Yuaner, Feng Ying. Research progress of TCM syndrome differentiation nursing for patients with lumbar disc herniation. Chin J Nurs, 2017, 52(5): 604-607.

16 Liu Ying, Han Ningzhen. Observation on the clinical efficacy of dialectical dietary therapy for constipation of bedridden patients. Sichuan Trad Chin Med, 2016, 34(6): 165-166. 